Journal of Computer Science 8 (8): 1372-1379, 2012

ISSN 1549-3636

(C) 2012 Science Publications

\title{
A Fuzzy Logic Based Power Control for Wideband Code Division Multiple Access Wireless Networks
}

\author{
${ }^{1,2} \mathrm{~N}$. Mohan and ${ }^{3,4}$ T. Ravichandran \\ ${ }^{1}$ Department of IT, AVS Engineering College, Salem, Tamilnadu, India \\ ${ }^{2}$ Anna University of Technology, Coimbatore, Tamilnadu, India \\ ${ }^{3}$ Hindustan Institute of Technology, Coimbatore, Tamilnadu, India \\ ${ }^{4}$ Anna University of Technology, Coimbatore, Tamilnadu, India
}

\begin{abstract}
Problem statement: Resource management is one of the most important engineering issues in 3G systems where multiple traffic classes are supported each being characterized by its required Quality of Service (QoS) parameters. Call Admission Control (CAC) is one of the resource management functions, which regulates network access to ensure QoS provisioning. Efficient CAC is necessary for the QoS provisioning in WCDMA environment. The effective functioning of WCDMA systems is influenced by the power control utility. Approach: In this study, we propose to design a fuzzy logic based power control for Wideband Code Division Multiple Access Wireless Networks. This proposed technique is aimed at multiple services like voice, video and data for multiclass users. The fuzzy logic technique is used to estimate the optimal admissible users group inclusive of optimum transmitting power level. This technique reduces the interference level and call rejection rate. Results: By simulation results, we demonstrate that the proposed technique achieve reduced energy consumption for a cell with increased throughput. Conclusion: The proposed technique minimizes the power consumption and call rejection rate.
\end{abstract}

Key words: Quality of Service (QoS), Call admission control (CAC), Wideband Code Division Multiple Access (WCDMA), Universal Mobile Telecommunication System (UMTS)

\section{INTRODUCTION}

Wideband Code Division Multiple Access (WCDMA) has emerged as the conventional air interface solution for $3 \mathrm{G}$ networks for instance Universal Mobile Telecommunication System (UMTS) and IMT-2000. Each signal broadcasted in the air interface is provided to the desired signal in the form of wideband noise as the WCDMA system is restricted in terms of interference. When more users are admitted, the quality of service (QoS) of the new user along with ongoing links are corrupted by the common interference exisitng among the links. Hence for the user to get benefitted with the requested QoS and also avoid the outage scenarios in the system caused by overloading, an admission control is taken into consideration. (Ayyappan and Kumar, 2010) For proper function, the modern WCDMA communication system such as UMTS requires a power control mechanism. The power control mechanism decreases the transmit power of every User Equipment (UE) to a value that realizes the required throughput and Block Error Ratio
(BLER). As the mutual interference created by the users is reduced, it also enhances the system wide data throughput (Laner et al., 2010a).

Power control in WCDMA: In view of the fact that the users share simultaneously the same transmission medium and thus interfere with one another, a power control is essential in Code Division Multiple Access (CDMA) or wideband CDMA (WCDMA) systems. If the transmitters use the same transmitter power, then the levels of several transmitter signals might vary considerably at the receiver due to channel variations. This is known as the near-far effect. In order to provide sufficient signal quality for all the users, the transmitter power have to be controlled which implies that the users must have sufficient Signal-to-Interference Ratios (SIRs) (Kabir et al., 2012).

The power control mechanism (PC) removes the near-far effect particularly in uplink direction and the received signal power arising from all users are also balanced. The different attenuations for instance path loss and shadowing owing to distance and shielding fact respectively and multipath fading are caused when signal

Corresponding Author: Mohan, N., Department of IT, AVS Engineering College, Salem, Tamilnadu, India 


\section{J. Computer Sci., 8 (8): 1372-1379, 2012}

is transmitted via a radio channel. Therefore, it is necessary to design the power control in WCDMA that can handle these attenuations.

The conventional fixed step PC algorithm is simple and needs interference level (SIR-based PC) and/or received signal power (S-based PC) measurements. These measured values are then compared with the targets and a decision on power control is sent over a feedback channel for the execution in the mobile unit.

The rapid modulations in the signal level at practical renewed rate cannot be handled by the fixed step algorithms and thus an adaptive step-size and predictive techniques have also been proposed. When multiple users contribute in one or multiple cells, the impact power control becomes a complicated issue. The power control commands of every user alter interference statistics monitored in other routes and it results in complex problem. This in turn results in SIR target optimization issues. This SIR target has to be adjustable as per the network load and transmitted data rates (Ali and Siddiqua, 2011).

Types of power control in WCDMA: Some of the power control methods followed in WCDMA is as follows (Laner et al., 2010a; 2010b):

Open Loop Power Control (OLPC): In the absence of dedicated channel, OLPC is used though closed loop power control gurantees the adequate tramission of power by the user in order to attain service needs for a specific dedicated link.

Closed Loop Power Control (CLPC): CLPC is utilized to assure whether the user transmits sufficient power to satisfy service needs. The two types of CLPC include Outer loop power control and Inner loop power control.

Outer Loop Power Control (OLPC): Based on the service requirement, OLPC is in charge for assigning the signal-to-interference ratio (SIR) target. In order to achieve a defined Block Error Ratio (BLER) by adjusting the Signal to Interference Ratio (SIR) target value, it achieves a control loop reaching from the user equipment to the core network. Because of the low required BLER, which cannot be measured accurately within the required response time of the control loop the above task bears some difficulties.

Inner Loop Power Control (ILPC): ILPC is mainly in charge to equalizing received SIR with SIR target for synchronizing the transmission power. The Fixed Step Size Power Control (FSPC) is the technique used in
UMTS. The receiver offers a transmit power control (TPC) command as per the received SIR for organizing transmission power of a mobile. Then the transmission power is adjusted by a fixed step size say $1 \mathrm{~dB}$ in general according to the TPC command.

Need for power control: Since the capacity of WCDMA is interference restricted, the capacity will decrease when the number of users in the WCDMA system is increased. In order to maximize the system capacity, it is vital to minimize the interference in the systems. The transmission power from additional users in the uplink is one of the vital sources of interference. A mobile nearby the base station may transmit excessive power, if the transmission power in the uplink is not appropriately regulated resulting in large interference to the further users connected to the same base station. This condition minimizes the capacity of the system and thus it is essential to organize the transmitted power (Laner et al., 2010b).

In CDMA, its performance gets directly affected since the system power control has a strong effect on the interference experienced by the receiver. The main characteristics of power control are compensation of fading channels and changes in the transmitted powers of interfering users. On the other hand, it might cause problems on the adaptation of equalizers (Buchbinder $e t$ al., 2010). A power control mechanism that assures that the received power levels from all User Equipments (UE) are identical at base station prevents the above issues (Laner et al., 2010a). The fundamental idea of power control mechanism is handling the minimum requirement for the Quality of Service (QoS) of the channel and to maximize the minimum Signal to Interference Ratio (SIR) in each of the channels in the CDMA system (Laner et al., 2010b). Therefore power control is seen as an important method to reduce mutual interference between the users, at the same time compensating for time varying propagation conditions. It also decreases the transmission power and maximizes the system capacity.

Problem identification and proposed work: In our previous study [pre], we have designed a new MAC protocol which consists of CAC algorithm with power control for multiple services like voice, video and data for multiclass users. It determines the optimum set of admissible users with optimum transmitting power level, so as to minimize the interference level and call rejection rate. It also contains an adaptive scheduling scheme to allocate optimum rate for each traffic queue and for minimizing the delay. The proposed scheme adapts for changing the traffic conditions based on estimation of future packet arrival rates. 
Thus as an extension to the previous work, we propose a Fuzzy based call admission control by predicting the load and power in the network. Our fuzzy based CAC is based on three main parameters such as Type of Service Request $\left(S_{R}\right)$, Total Load Factor $\left(L_{D L}\right)$ and the Total Power $\left(\mathrm{P}_{\mathrm{T}}\right)$. Based on these parameters, we form a set of fuzzy decisions denoting the acceptability of the new call whether it is Strongly Rejected, Weakly rejected, Weakly Accepted, or Strongly Accepted.

Related work: Rao et al. (2011) have proposed an adaptive power control mechanism for multimedia traffic in WCDMA networks. In their proposed algorithm, they have used two latest Transmit Power Control (TPC) commands to compute the Adaptive Factor based on a predefined Adaptive Control Factor (ACF). They have also introduced another parameter called Power Determining Factor (PDF) based on the data traffic rate in order to determine the power. Based on their new parameter, the power was increased or decreased. Also, depending on the traffic rate, their PDF factor was updated such that if the observed traffic rate was high, then they would increase the parameter and subsequently increased the power. Suppose, if the traffic rate was low, then their parameter would be decreased and correspondingly the power too.

Aguero et al. (2009) have presented new methods to enhance control regarding the strong non-linear effect. Therefore they have proposed adaptive control architecture for inner loop power control, containing three degrees of freedom. Their three degrees of freedom were utilized to deal with errors that is caused due to channel/interference variations, quantization of the power increments and saturation of the power increments. They included a adaptation mechanism to their proposed technqiue for tracking non-stationary channel gain and interference models. The main drawback of this approach is concerned with the transmission of channel interference model information from base station to the mobile unit.

Laner et al. (2010b) have analyzed the uncoded BER parameter for enhancing uplink OLPC algorithm. They performed live experiments which showed that the actual implemented algorithm converged gradually. They justified their approach based on QoS estimated by CRC and since the uncoded Bit Error Ratio (BER) contains information regarding the QoS. Also this paramater is used to enhance the convergence speed of their OLPC. They have also presented a statistical model of the control path of the OLPC that takes uncoded BER information into consideration
Rachidi et al. (2004) have presented a QoS aware mechanism for power control and Handoff in 3G WCDMA networks. They have also presented two mechanisms named Quality of Service aware Power Control (QaPC) and QoS aware prioritization of Handoffs (QaHO) which were based on the class of service, bit rate and Service Degradation Descriptor (SDD) as enabling QoS parameters. They have also obtained the numerical results using a WCDMA and UMTS compatible test bed which showed that their proposed QoS aware mechanism significantly improved the QoS contract upholding for premium mobile users, as well as increased resource utilization, while improving the acceptance of soft handoffs.

\section{MATERIALS AND METHODS}

Call Admission Control (CAC) parameters: Our CAC is based on three main parameters such as Type of Service Request $\left(\mathrm{S}_{\mathrm{R}}\right)$, Total Load Factor $\left(\mathrm{L}_{\mathrm{DL}}\right)$ and the Total Power $\left(\mathrm{P}_{\mathrm{T}}\right)$.

Computation of Type of Service Request $\left(S_{R}\right)$ : The type of Service $\left(S_{R}\right)$ taken in to account are of two types namely Real Time (RT) and Non Real Time (NRT). The Real Time services are provided with a high priority when compared with those of Non Real Time services Eq. 1:

$S_{R}=\left\{\begin{array}{l}1, \text { ForRT } \\ 0, \text { For NRT }\end{array}\right.$

Computation of Load Factor $\left(\mathbf{L}_{\mathrm{DL}}\right)$ : The computation of the downlink existing load factor $\left(\mathrm{L}_{\mathrm{DL}}\right)$ is given in Eq. 2 (Ayyappan and Kumar, 2010):

$L_{D L}=\sum_{i=1}^{N} B_{R} \frac{V_{F}^{i} \frac{E_{b}}{N_{D}}}{C_{R}}\left[\left(1-\varphi_{a v}\right)+T_{i}\right]$

Where:

$\mathrm{B}_{\mathrm{R}} \quad=$ The bit rate of ith user

$\mathrm{V}_{\mathrm{F}}^{\mathrm{i}} \quad=$ The voice activity factor of ith user

$\mathrm{E}_{\mathrm{b}} / \mathrm{N}_{\mathrm{D}}=$ The bit energy to noise density ratio of ith user

$\mathrm{C}_{\mathrm{R}}=$ The chip rate

$\phi_{\mathrm{av}}=$ The average orthogonality factor of the cell

$\mathrm{T}_{\mathrm{i}} \quad=$ The total interference

$\mathrm{N}=$ The number of users

Computation of Total Power $\left(\mathbf{P}_{\mathrm{T}}\right)$ : The downlink power for $\mathrm{k}^{\text {th }}$ user can be expressed using Eq. 3 as follows (Khalek et al., 2011): 
$\mathrm{P}_{\mathrm{R}}^{\mathrm{k}}=\mathrm{L}_{\mathrm{P}}^{\mathrm{k}} * \mathrm{P}_{\mathrm{MS}}^{\mathrm{k}}$

Where:

$P_{\mathrm{R}}^{k}=$ The downlink power of $\mathrm{k}^{\text {th }}$ user

$\mathrm{L}_{\mathrm{P}}^{\mathrm{k}}=$ The estimated path loss between $\mathrm{k}^{\text {th }}$ MS and its serving BS $\begin{aligned} \mathrm{P}_{\mathrm{MS}}^{\mathrm{k}}= & \text { The transmit power allocated to } \mathrm{k}^{\text {th }} \text { MS by its } \\ \text { serving BS } & \end{aligned}$

The total power of $\mathrm{N}$ number of users served by a single BS is given by the summation of each individual power of all the users such that Eq. 4 :

$\mathrm{P}_{\mathrm{T}}=\sum_{\mathrm{k}=1}^{\mathrm{N}} \mathrm{L}_{\mathrm{P}}(\mathrm{k}) * \mathrm{P}_{\mathrm{MS}}(\mathrm{k})$

Fuzzy logic based Call Admission Control (CAC):

Basic concepts of fuzzy logic: Fuzzy logic is a problem solving control system method that offers itself to implementation in systems. It can be implemented in hardware, software, or a combination of both. Fuzzy logic provides a simple way to arrive at a definite conclusion based upon vague, ambiguous, imprecise, noisy, or missing input information. Fuzzy logic is a form of many valued logic and it deals with reasoning that is fairly accurate rather than fixed and exact.

Fuzzy congestion controller (FCC) is said to be Fuzzy logic controller (FLC). The process involved in FLC includes the selection of adequate mathematical representations such as t-norm, defuzzification, operators, fuzzy implication functions and shapes of membership functions between the rich among a rich set of candidates. This method of choosing the specific operators and functions modulates the non-linear inputoutput relationship of FLC. However the results have shown that these effects can also be attained by altering the rule base appropriately. Hence a computationally lighter and well learned operators and functions are chosen in practical applications. Also the preferred behavior of FLC can be attained by modifying linguistic rules.

For the implementation of the FCC, the most widely used and computationally lighter methods are as follows:

- Singleton fuzzification

- t-norm algebraic product for the mathematical representation of the connective "and"

- Larsen's product rule of implication

- Sub-product compositional rule of inference

- Weighted mean of maximums defuzzification
Fuzzification: Fuzzification is defined as the mapping from a real valued point to a fuzzy set. The FLC system in this study receives the required information as the inputs of the FLC and the fuzzification module converts inputs into fuzzy linguistic variable inputs.

Two major reasons for the choice of fuzzy logic are:

- Absence of clear boundaries between the normal and abnormal events

- The abrupt separation of normality and abnormality are smoothened by the fuzzy logic rules. A membership function is a mathematical formation of representing a fuzzy set

Rule definition: A membership function which characterizes a fuzzy set $\mathrm{A}$ in $\mathrm{x}$ can be implemented easily using conditional statements. If an antecedent in a fuzzy statement is true to some degree of membership then the consequent is also true to that same degree:

- Rule structure: If antecedent then consequent

- The rule: If one variable is low and one variable is high then output will be benevolent else it will be malevolent.

On applying a set of fuzzy rules based on the linguistic values of its attributes a case or an object can be classified in a fuzzy classification system. The rule is applied to the number given by the antecedent. This rule has a weight which is numbered between 0 and 1 . Initially the antecedent is evaluated which fuzzifies the input and applies any necessary fuzzy operators. Then the result is applied to the consequent which is known as inference. A set of fuzzy rules related to specific classification problem need to be found for building a fuzzy classification system. This is considered as the most difficult task.

We will now describe our methodology of fuzzy logic based call admission control for WCDMA networks. The three most important variables taken in to account are Type of Service Request $\left(S_{R}\right)$, Total Load Factor $\left(\mathrm{L}_{\mathrm{DL}}\right)$ and the Total Power $\left(\mathrm{P}_{\mathrm{T}}\right)$. With fuzzy logic, we assign grade values to our three variables. Our fuzzy set therefore consists of three fuzzy variables:

$$
\text { Fuzzy set }=\left\{\mathrm{S}_{\mathrm{R}}, \mathrm{L}_{\mathrm{DL}}, \mathrm{P}_{\mathrm{T}}\right\}
$$

Fuzzy logic implements human experiences and preferences via membership functions and fuzzy rules. In this work, the fuzzy if-then rules consider the parameters: Type of Service Request $\left(S_{R}\right)$, Total Load Factor $\left(\mathrm{L}_{\mathrm{DL}}\right)$ and the Total Power $\left(\mathrm{P}_{\mathrm{T}}\right)$. 
The fuzzy logic uses three input variables and one output variable. The inputs are fuzzified, implicated, aggregated and defuzzified to get the output.

The inputs parameters are:

$\mathrm{S}_{\mathrm{R}}$ : Type of service request

(Real time or Non-real time)

$\mathrm{P}_{\mathrm{T}}$ : Total Power

$\mathrm{L}_{\mathrm{DL}}$ : Available load

The linguistic variables associated with the input variables are Low $(\mathrm{L})$ and High $(\mathrm{H})$.

The corresponding fuzzy linguistic term set is:

$\mathrm{F}\left(\mathrm{S}_{\mathrm{R}}\right):\{$ High-RT, Low-NRT $\}$

$\mathrm{F}\left(\mathrm{P}_{\mathrm{T}}\right):\{$ Low, High $\}$

$\mathrm{F}\left(\mathrm{L}_{\mathrm{DL}}\right):\{$ Low, High $\}$

The output variables use four linguistic variables SR, WR, WA and SA, where SR denotes acceptability of the new call is Strongly Rejected, WR denotes the acceptability of the new call is Weakly rejected, WA denotes the acceptability of the new call is Weakly Accepted and SA denotes the acceptability of the new call is Strongly Accepted.

The output linguistic variable, denoting the acceptability of the new call is:

F (D): \{Strongly Rejected, Weakly rejected, Weakly Accepted, Strongly Accepted $\}$.

Defuzzification: Mapping from a space of fuzzy control action defined over an output universe of discourse into a space of non fuzzy (crisp) control act ions is known as the defuzzification. A crisp control action that best represents the possibility distribution of an inferred fuzzy control action is produced by the defuzzification strategy.

Center Of Area (COA): Here, the center of gravity of the output membership function is used for selecting the output crispy value:

$$
\mathrm{U}_{\mathrm{o}}=\frac{\int \mathrm{w} \mu(\mathrm{w}) \mathrm{dw}}{\int \mu(\mathrm{w}) \mathrm{dw}}
$$

This defuzzification method is used in our fuzzy based rate control.

\section{RESULTS AND DISCUSSION}

Simulation model and parameters: This section deals simulation of our proposed Fuzzy Logic based Power Control (FLPC) in WCDMA cellular networks for admissible and non-admissible transmission scenarios.

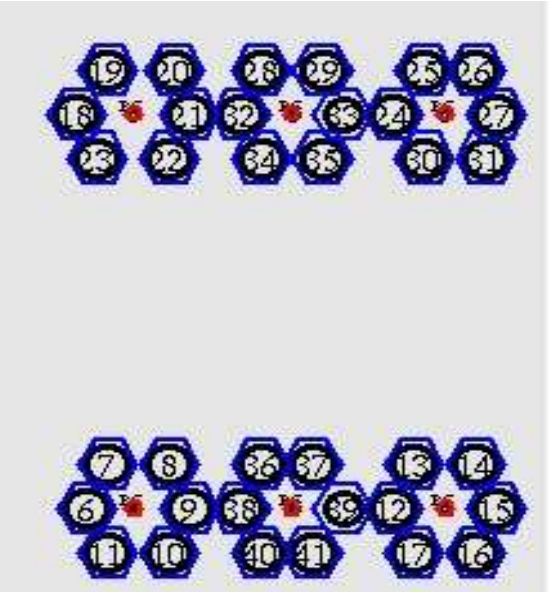

Fig. 1: Simulation topology

\begin{tabular}{|c|c|c|c|c|}
\hline $\begin{array}{l}\text { Fuzzy } \\
\text { rule }\end{array}$ & $\begin{array}{l}\text { Type of service } \\
\text { request }\left(S_{R}\right)\end{array}$ & $\begin{array}{l}\text { Total load } \\
\text { factor }\left(L_{D L}\right)\end{array}$ & $\begin{array}{l}\text { Total } \\
\text { power }\end{array}$ & $\begin{array}{l}\text { Fuzzy } \\
\text { Decision (D) }\end{array}$ \\
\hline 1 & High & Low & Low & SA \\
\hline 2 & High & Low & High & WA \\
\hline 3 & High & High & Low & WR \\
\hline 4 & High & High & High & SR \\
\hline 5 & Low & Low & Low & SA \\
\hline 6 & Low & Low & High & WA \\
\hline 7 & Low & High & Low & WR \\
\hline 8 & Low & High & High & SR \\
\hline
\end{tabular}

Table 2: Simulation parameters

\begin{tabular}{ll}
\hline Number of nodes & 36 \\
\hline No. of cells & 6 \\
Users per cell & 6 \\
Slot Duration & $2 \mathrm{~m} \mathrm{sec}$ \\
SINR threshold & 5 \\
Frame Length & $3 \mathrm{slots}$ \\
Txpower & $0.66 \mathrm{w}$ \\
RxPower & $0.395 \mathrm{w}$ \\
Routing protocol & AODV \\
Speed of mobile & $25 \mathrm{~m} \mathrm{sec}^{-1}$ \\
Traffic model & $\mathrm{CBR}$ \\
Initial energy & $4.1 \mathrm{~J}$ \\
\hline
\end{tabular}

The simulation tool used in NS2 is the general purpose simulation tool which offers discrete event simulation of user defined networks. During simulation, mobile nodes travel in $600 \times 600 \mathrm{~m}$ rectangular region at $50 \mathrm{sec}$ simulation time. The initial allocations and mobility of nodes are acquired using the random waypoint (RWP) model of NS2. The entire nodes possess the transmision range of $250 \mathrm{~m}$. Our proposed scheme is compared with QAHO proposed in (Rachidi et al., 2004). The experimental scenario for our experiment is shown in Fig. 1.

The fuzzy rules using SR, LDL and PT are shown in Table 1. The simulation parameters are summarized in Table 2. 
J. Computer Sci., 8 (8): 1372-1379, 2012
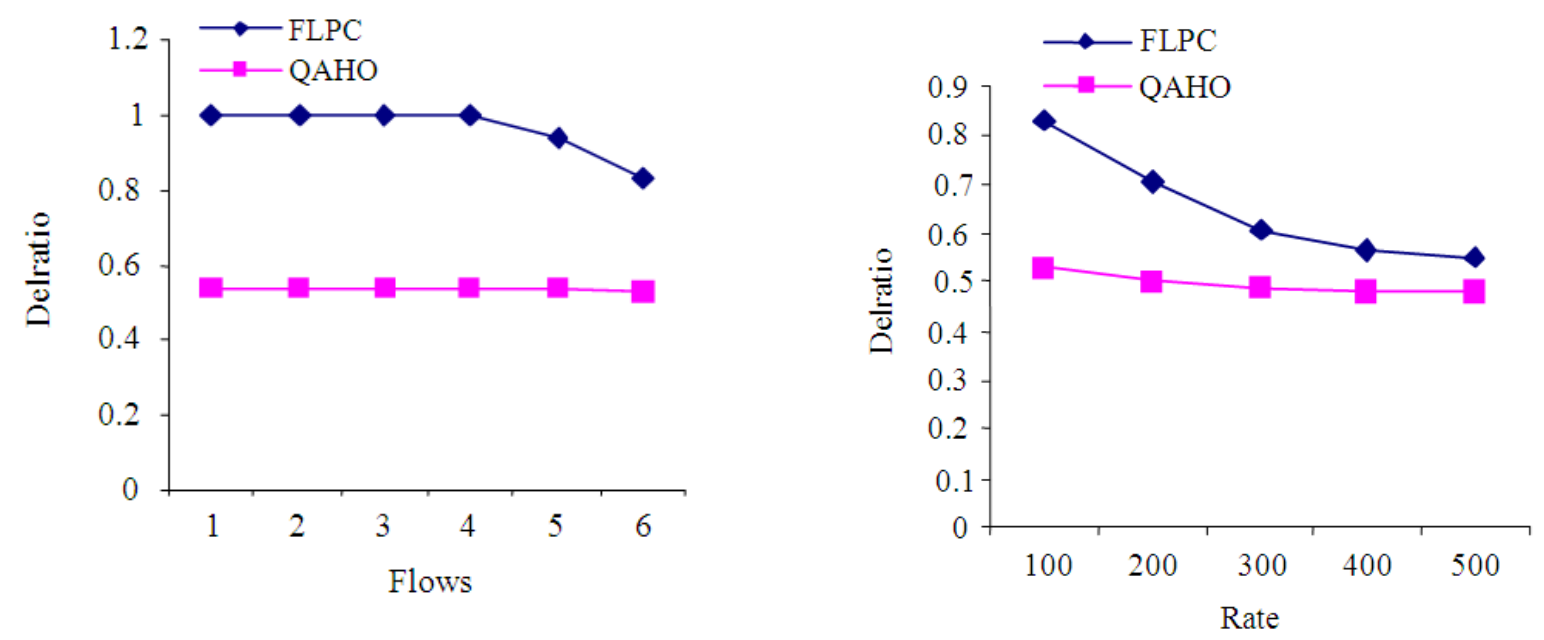

Fig. 2: Flows Vs Delratio

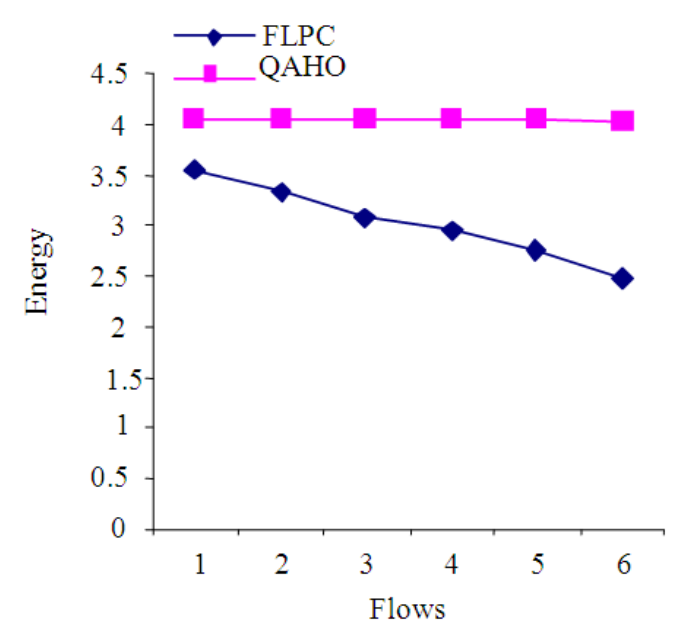

Fig. 3: Flows Vs energy

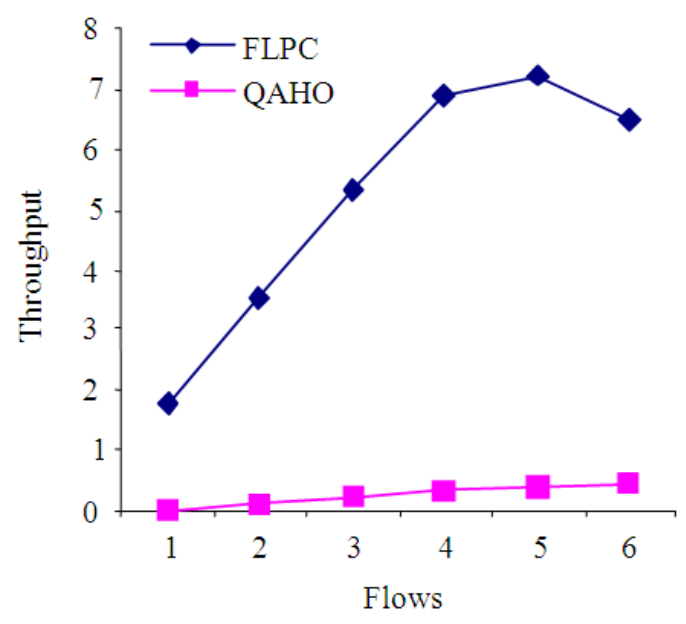

Fig. 5: Rate Vs Delratio

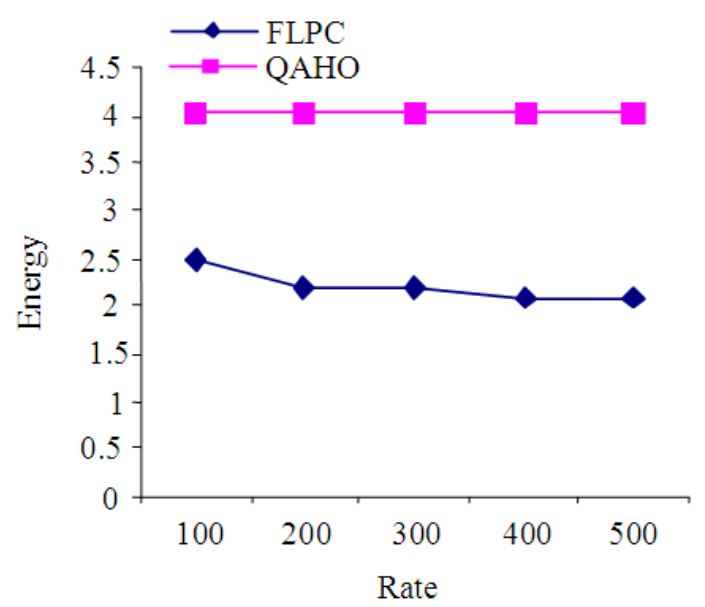

Fig. 6: Rate Vs energy

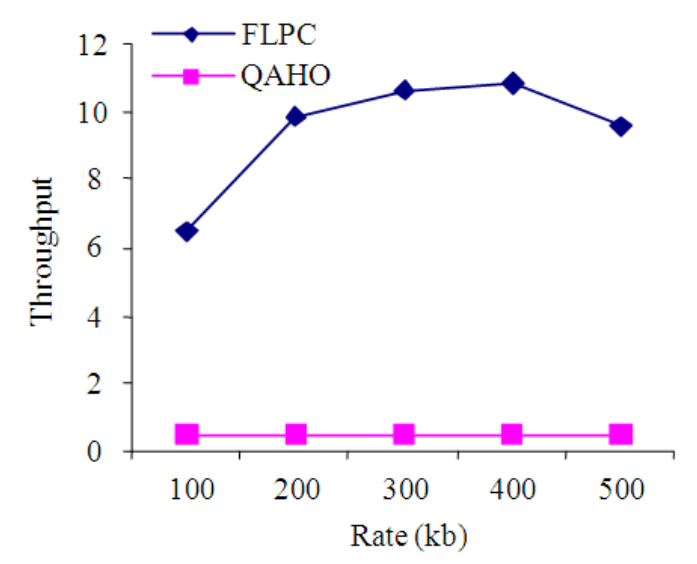

Fig. 4: Flows Vs throughput

Fig. 7: Rate Vs throughput 1377 


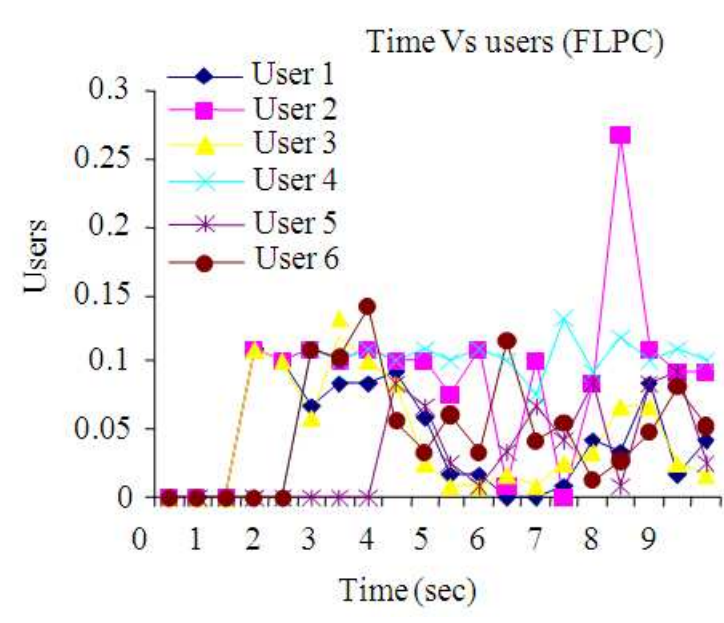

Fig. 8: Throughput for Users (FLPC)

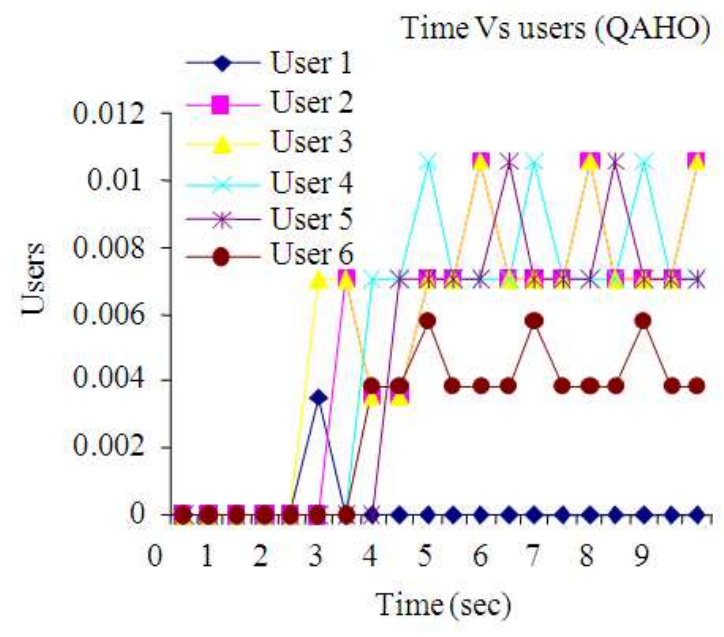

Fig. 9: Throughput for Users (QAHO)

\section{Simulation results:}

Based on number of users: In our first experiment, we vary the number of users as $1,2,3 \ldots 6$.

Figure 2 gives the delivery ratio calculated in both the schemes. From the figure, it is clear that our FLPC scheme have more delivery ratio, when compared with the QAHO scheme.

From Fig. 3, we can observe that our proposed FLPC has low average power consumption, when compared with the QAHO.

Figure 4 shows the average throughput measured. From the figure, it is apparent that our FLPC scheme attains more throughputs when compared to the QAHO scheme.

Based on rate: In our second experiment, we vary the data sending rates as $100,200 \ldots 500 \mathrm{~kb}$.
Figure 5 gives the delivery ratio calculated in both the schemes. From the figure, it is clear that our FLPC scheme have more delivery ratio, when compared with the QAHO scheme.

From Fig. 6, we can observe that our proposed FLPC has low average power consumption, when compared with the QAHO.

Figure 7 shows the average throughput measured. From the figure, it is apparent that our FLPC scheme attains more throughputs when compared to the QAHO scheme.

Based on time: In our next experiment, based on time we measure the throughput of each user in both the schemes.

Figure 8 shows the performance of our FLPC scheme for varying time with different users.

Figure 9 shows the performance of QAHO scheme for varying time with different users.

\section{CONCLUSION}

We have studied the fuzzy based CAC scheme for wideband CDMA cellular system for fulfilling the issues existing in CAC owing to user mobility, restricted radio spectrum, heterogenous and dynamic nature of multimedia traffic and QoS constraints and its performance is analyzed. In this study, we proposed a Fuzzy based CAC by measuring the total power and load in the network. Our fuzzy based CAC is based on three parameters such as Type of Service Request $\left(S_{R}\right)$, Total Load Factor $\left(\mathrm{L}_{\mathrm{DL}}\right)$ and the Total Power $\left(\mathrm{P}_{\mathrm{T}}\right)$. Then the fuzzy rules are formed, which take the value of the above three parameters as input. Then the output is given in the form of fuzzy variables which indicates the acceptability of the new call. Then defuzzification is done to get a crisp value of the output. By simulation experiments, we have shown that athe proposed algorithm achieve reduced energy consumption for a cell with increased throughput.

\section{REFERENCES}

Aguero, J.C., G.C. Goodwin, K. Lau, M. Wang and E.I. Silva et al., 2009. Three-degree of freedom adaptive power control for CDMA cellular systems. Proceedings of the IEEE Global Telecommunications Conference, Nov. 30-Dec. 4, IEEE Xplore Press, Honolulu, HI, pp: 1-6. DOI: 10.1109/GLOCOM.2009.5425906

Ali, T. and P. Siddiqua, 2011. Power control in CDMA/WCDMA systems using neural network based noise-suppression technique. Int. J. Comput. Sci. Emerging Technol. 
Ayyappan, K. and R. Kumar, 2010. QoS based capacity enhancement for WCDMA network with coding scheme. Int. J. VLSI Design Commun. Syst., 1: 10-19.

Buchbinder, N., L. Lewin-Eytan, I. Menache, J. Naor and A. Orda, 2010. Dynamic power allocation under arbitrary varying channels - the multi-user case. Proceedings of the 29th conference on Information communications, (INFOCOM' 10), ACM Press, USA., pp: 2402-2410.

Khalek, A.A., L. Al-Kanj, Z. Dawy and G. Turkiyyah, 2011. Optimization models and algorithms for joint uplink/downlink UMTS radio network planning with sir-based power control. IEEE Trans. Vehicular Technol., 60: 1612-1625. DOI: 10.1109/TVT.2011.2132745

Kabir, P., M.H. Shafinia, F. Marvasti and P. Pad, 2012. Capacity bounds of finite dimensional CDMA systems with fading/near-far effects and power control. Comput. Sci. Inform. Theory.

Laner, M., P. Svoboda and M. Rupp, 2010a. On the influence of UMTS power control on the linklevel error statistics. Vienna University of Technolog Austria.
Laner, M., P. Svoboda and M. Rupp, 2010b. Outer-loop power control in a live UMTS network: Measurement, analysis and improvements. Proceedings of the 4th International Symposium on Communications, Control and Signal Processing, Mar. 3-5, IEEE Xplore Press, Limassol, pp: 1-5. DOI: $10.1109 /$ ISCCSP.2010.5463465

Rachidi, T.E., A.Y. Elbatji, M. Sebbane and H. Bouzekri, 2004. QoS-aware power control and handoff prioritization in 3G WCDMA networks. proceedings of the IEEE Wireless Communications and Networking Conference, Mar. 21-25, IEEE Xplore Press, pp: 665-670. DOI: 10.1109/WCNC.2004.1311265

Rao, S.P.V.S., S.V. Chalam and D.S. Rao, 2011. A dynamic MAC protocol with adaptive power control for WCDMA networks. Int. J. Distributed Parallel Syst., 2: 105-114. 